1 Universidade de Pernambuco (UPE), Faculdade de Enfermagem Nossa Senhora das Graças (Fensg), Grupo de Pesquisadores da África e América Latinas (Graal) Recife (PE), Brasil.

rejane.ferreira@upe.br

2 Fundação de Amparo à Ciência e Tecnologia do Estado de Pernambuco (Facepe) - Recife (PE), Brasil.

jpreisbraga@hotmail.com

3 Instituto de Medicina Integral Professor Fernando Figueira (Imip) - Recife (PE), Brasil.

jfpmoura@hotmail.com

4 Instituto de Medicina Integral Professor Fernando

Figueira (Imip) - Recife (PE), Brasil.

jurema@imip.org.br

\section{Continuidade Assistencial a mulheres com câncer de colo de útero em redes de atenção à saúde: estudo de caso, Pernambuco}

\author{
Care Continuity to women with cervical cancer in health care \\ networks: case study, Pernambuco \\ Maria Rejane Ferreira da Silva', João Paulo Reis Braga², José Fernando do Prado Moura², Jurema \\ Telles de Oliveira Lima 4
}

RESUMO O objetivo do estudo foi analisar a continuidade da assistência a mulheres com câncer de colo uterino. Estudo de caso qualitativo, em redes de saúde. Realizaram-se entrevistas com roteiro semiestruturado e técnica de análise narrativa de conteúdo. As mulheres perceberam barreiras no diagnóstico, no tratamento e na acessibilidade entre níveis de atenção. Observaram comunicação informal entre especialistas; limitações na comunicação entre níveis de atenção; inexistência de contrarreferência; e que o vínculo paciente-serviço era maior na esfera da oncologia. A falta de contrarreferência dificultou a acumulação de conhecimento e a coordenação do cuidado pela Atenção Básica.

PALAVRAS-CHAVE Continuidade da assistência ao paciente. Acesso aos serviços de saúde. Atenção Primária à Saúde. Estudos de casos. Promoção da saúde.

ABSTRACT The aim of the study was to analyze the continuity of care for women with cervical cancer. Qualitative study of case, in health networks. Interviews were performed with semi-structured script and technique of content's narrative analysis. Women perceived barriers in the diagnosis, treatment and accessibility between levels of care. They observed informal communication between specialists; limitations in the communication between levels of care; absence of counter-reference; and that the patient-service relationship was greater in the oncology sphere. The lack of counter-reference hindered the accumulation of knowledge and coordination of care for Primary Care.

KEYWORDS Continuity of patient care. Health services accessibility. Primary Health Care. Case studies. Health promotion. 


\section{Introdução}

A estruturação de Redes Integradas de Serviços de Saúde (Riss) tem sido a principal estratégia utilizada, nas últimas três décadas, em meio às reformas dos sistemas de saúde de quase todos os países latino-americanos, entre eles, o Brasil. Uma Riss representa a etapa final do processo de integração assistencial. Seus objetivos intermediários são o acesso, a coordenação clínica e a continuidade da assistência, e os objetivos finais são a equidade de acesso, a qualidade da assistência e eficiência. Neste sentido, as ditas reformas objetivaram melhorar o acesso, aperfeiçoar a coordenação e a Continuidade Assistencial (CA), e aumentar a eficiência nos cuidados ofertados à população (MENDES, 2011; SHORTELL ET AL., 2000).

O conceito de CA tem sido definido de diversas formas na literatura científica, podendo envolver várias dimensões da rede assistencial. No entanto, ainda não há uma definição consensual entre os estudiosos do tema. Não obstante, para a maioria dos autores, a CA está diretamente relacionada com a coordenação da atenção (busca pela integração entre os serviços), com a transferência da informação e com a relação interpessoal entre pacientes e cuidadores.

Neste estudo, o conceito utilizado foi o proposto por Reid, Haggerty e McKendry (2002, P. 5), que diz que a CA pode ser analisada de acordo com o "grau de coerência e união das experiências na atenção, percebida pelos usuários ao longo do tempo", e que essa assistência deve estar em conformidade com as "necessidades de saúde e o contexto pessoal" de cada paciente. Desta forma, a CA deve ser percebida e expressar o ponto de vista do paciente a respeito dos serviços que ele acessou em sua trajetória na Riss. Foram identificados três tipos distintos de CA: (a) a continuidade da gestão clínica, que está relacionada com a coordenação da atenção e com o fornecimento de diferentes tipos de cuidados complementares ao longo do tempo; (b) a continuidade da informação, relacionada com a disponibilidade e a utilização das informações sobre o paciente em ocorrências anteriores; e (c) a continuidade da relação, que se refere ao nível de relação estabelecida entre o paciente e o médico que assumiu a responsabilidade do cuidado desse usuário ao longo do tempo.

Dentro do conceito proposto por Reid, Haggerty e McKendry (2002) existem subdimensões para os três tipos de CA. Os tipos e suas subdimensões de CA, tais como apresentadas na figura 1, representam as categorias teóricas que serão analisadas neste estudo e que orientaram a formulação do roteiro de entrevistas.

A 'continuidade de gestão' clínica se expressa em três dimensões: (a) a acessibilidade entre os níveis assistenciais, que se caracteriza pela oportunidade de transposição de nível, de acordo com a necessidade e no tempo oportuno; (b) a consistência do cuidado ou coerência da atenção, que é definida como a percepção, por parte dos pacientes, de que exista coerência nos objetivos e tratamentos realizados por diferentes serviços (provedores); e (c) a flexibilidade nos planos de cuidado, definida como a percepção do paciente de que sua atenção se adapta às mudanças de suas necessidades e circunstâncias.

A 'continuidade da informação' está subdividida em: (a) a transferência da informação, que é a percepção do usuário de que cada serviço (provedor) tem acesso à informação sobre a atenção prestada anteriormente e ao desenvolvimento de sua doença; e (b) o conhecimento acumulado, que está relacionado não só à percepção do paciente, mas também ao profissional que o atendeu se este conhece seus valores e preferências, e se isto influencia no planejamento do tratamento mais apropriado.

$\mathrm{E}$, finalmente, na 'continuidade de relação' existem duas subdivisões: (a) o vínculo entre o paciente e o serviço de saúde, que tem duração variável de acordo com o tipo de 
assistência demandada (episódio agudo ou enfermidade crônica de longa duração); e (b) a estabilidade e a consistência do profissional, que estão vinculadas à possibilidade de o paciente ser atendido pelos mesmos profissionais (provedores), caracterizando-se pela responsabilidade e pela confiança entre usuário e equipe, ainda que não se estabeleçam relações em longo prazo (REID; HAGGERTY; MCKENDRY, 2002).

Figura 1. Tipos e dimensões da continuidade da atenção

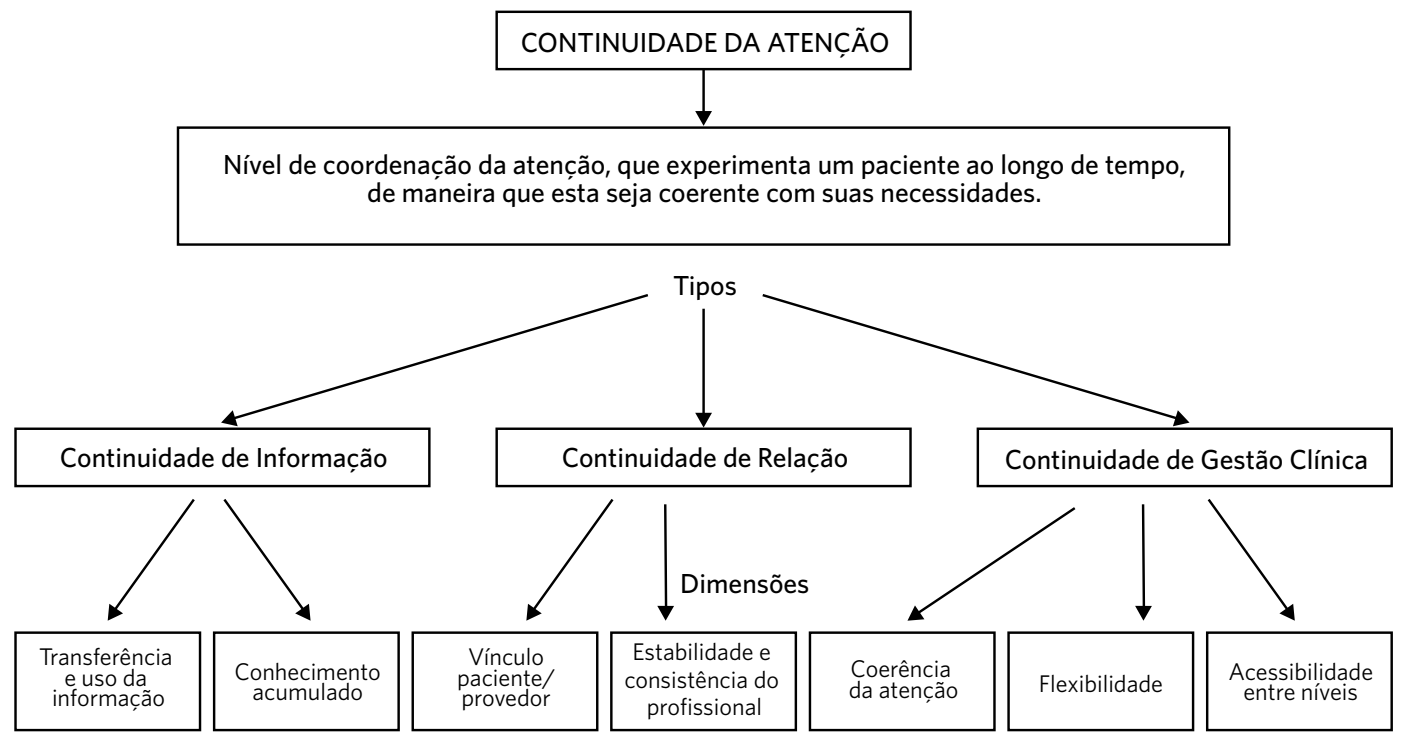

Fonte: Reid, Haggerty e McKendry (2002).

Atualmente, a CA tem relevância na análise dos cuidados prestados a pacientes de doenças crônicas como o câncer de colo do útero, dentro das Riss, em virtude da ampla gama de aspectos que o conceito considera. Não obstante, a literatura científica demonstra uma forte escassez de estudos sobre essa temática, tanto que, na revisão bibliográfica, não foram encontrados estudos a respeito da CA em pacientes portadoras de neoplasia maligna no colo do útero.

O câncer cervical, ou de colo de útero, é o quarto tipo mais frequente de câncer em mulheres do mundo todo (WHO, 2012). E aproximadamente $70 \%$ dos casos são diagnosticados em países em desenvolvimento (STEWART; WILD, 2014). No Brasil, a neoplasia de colo do útero é o segundo tipo de câncer mais prevalente na população feminina, com estimativas de cerca de 15 mil novos casos e de aproximadamente cinco mil mortes por ano (INCA, 2014). Particularmente na região Nordeste, o câncer do colo uterino figura entre os mais incidentes no sexo feminino, e Pernambuco está entre os estados com maior incidência da doença (INCA, 2014).

O tratamento de mulheres com câncer cervical exige a atuação de equipes multidisciplinares, nos três níveis assistenciais da rede e por um considerável período de 
tempo. Assim, a análise da CA nesses casos torna possível conhecer o atual estágio de integração entre os diferentes níveis de cuidados de determinada rede de serviços de saúde, e pode, ainda, fornecer elementos que permitam compará-las a outras redes assistenciais (BRITO; OLIVEIRA; SILVA, 2012). O presente artigo tem como objetivo analisar a continuidade da atenção a mulheres portadoras de câncer de colo do útero.

\section{Métodos}

A pesquisa adotou como desenho um estudo de caso com abordagem qualitativa, para coletar e examinar evidências sobre a continuidade da assistência à saúde vivenciada por mulheres que passaram por tratamentos em redes de saúde de Pernambuco.
Utilizou-se como condição traçadora o câncer de colo do útero. Essa neoplasia foi selecionada mediante os seguintes critérios: ser enfermidade prevalente entre o grupo de mulheres, requerer a intervenção de diferentes níveis assistenciais (primeiro nível e atenção especializada) e porque a assistência à usuária demanda o uso de protocolos. Foram selecionadas três das quatro redes de atenção oncológica planejadas para o atendimento ao doente com câncer, cujas sedes eram em Recife (PE), Caruaru (PE) e Garanhuns (PE), estas últimas por serem mais próximas da capital e apresentarem mais factibilidade. Em todas as sedes, foram selecionados dois casos: um do município-sede e outro de um município da macrorregião de cada sede. A composição final dos casos está apresentada na tabela 1.

Tabela 1. Tipos e dimensões da continuidade da atenção

\begin{tabular}{lrrrrr}
\hline Fontes & Recife & Caruaru & Garanhuns & Total \\
\hline Usuárias & Câncer de colo de útero & 2 & 2 & 2 & 6 \\
\multirow{2}{*}{$\begin{array}{l}\text { Profissionais } \\
\text { APS }\end{array}$} & 3 & 1 & 2 & 6 \\
\hline Total & Especializada & 4 & $\mathbf{5}$ & $\mathbf{7}$ & $\mathbf{2 1}$ \\
\hline \multirow{2}{*}{ Prontuários } & APS $^{\star}$ & $\mathbf{9}$ & 0 & 0 & 2 \\
& Especializado & 4 & 1 & 4 & 9 \\
\hline
\end{tabular}

Fonte: Elaboração própria.

*APS - Atenção Primária à Saúde.

Foram feitas coleta de dados nos prontuários das unidades e entrevistas com todas as usuárias e médicos por elas indicados, com os quais foram consultadas.

A seleção das mulheres foi feita empregando-se os seguintes critérios: mulheres maiores de 18 anos, com diagnóstico confirmado há, no mínimo, um ano; e com estabilidade física e mental. Foram identificadas usuárias que necessitaram da atenção de diversos níveis assistenciais, com e sem complicações associadas.

Objetivando melhorar a compreensão acerca da temática, foram revisados os 
prontuários das mulheres encontrados nas unidades assistenciais onde receberam atendimento. Foram realizadas entrevistas semiestruturadas, com roteiro específico dirigido às seis usuárias e aos dez profissionais de saúde responsáveis por essas pacientes na rede. As entrevistas foram realizadas individualmente, nos domicílios das usuárias, nos locais de trabalho dos profissionais ou nos locais onde as usuárias fizeram tratamento, no período de dezembro de 2013 a julho de 2014. As informações dos profissionais complementaram as fornecidas pelas usuárias e, posteriormente, todos os dados foram triangulados com as informações obtidas através da análise dos prontuários clínicos.

As entrevistas, após serem gravadas e transcritas, foram analisadas usando o software de codificação de texto Atlas Ti (v. 6.0). A análise do conteúdo das entrevistas foi realizada a partir das categorias pré-estabelecidas, com base no marco teórico do projeto (figura 1), através da análise narrativa de conteúdo (BARDIN, 2009). Em seguida, foi feita uma nova classificação dos conteúdos para comparação entre as redes, estabelecendo as principais semelhanças ou diferenças entre elas. Os resultados foram categorizados de acordo com o referencial teórico, dentro dos grupos principais estabelecidos para a CA: continuidade da gestão clínica, continuidade da informação, e continuidade da relação e suas respectivas dimensões (HAGGERTY ET AL., 2003).

Para garantir o anonimato dos entrevistados e a confidencialidade das informações, atribuíram-se identificações fictícias aos entrevistados com o uso de letras maiúsculas e números para cada caso, destacando-se, porém, em cada citação, a localidade da rede. A pesquisa foi aprovada pelo Comitê Nacional de Ética em Pesquisa (CAAE 07359713.3.0000.5207) e teve a autorização das secretarias de saúde dos municípios, bem como da Secretaria Estadual de Saúde de Pernambuco.

\section{Resultados}

Os resultados do estudo evidenciaram vários elementos que influenciam as distintas dimensões da CA de mulheres com câncer de colo do útero nos serviços assistenciais de Pernambuco. Nas três redes de saúde analisadas, foram observados barreiras e facilitadores da CA. Porém, nenhuma das redes demonstrou oferecer um serviço plenamente satisfatório às usuárias, posto que, embora para todas elas tenha sido possível acessar e cumprir o tratamento preconizado, em alguns casos, isto se deu tardiamente.

Os problemas na CA das mulheres com câncer de colo do útero se evidenciaram principalmente nas dificuldades de acessibilidade aos distintos níveis assistenciais, que apareceram desde a fase pré-diagnóstica e continuaram acontecendo durante o seguimento. Em todo o relatório, foram descritos problemas comuns às redes de Recife (PE), Caruaru (PE) e Garanhuns (PE), mas também emergiram barreiras que se manifestam na individualidade de cada rede.

\section{Continuidade da gestão clínica}

$\mathrm{Na}$ análise dos discursos relacionados com a continuidade da gestão clínica na assistência de mulheres com câncer de colo do útero, emergiram problemas como o descumprimento de normas para a prevenção e o diagnóstico precoce do câncer, aliados ao fato de que quase todos os médicos das três redes não fizeram o referenciamento correto da paciente, nem seguiram os protocolos estabelecidos para suspeita de câncer (apesar d'eles existirem). Em Recife (PE), destacou-se o fato de duas pacientes terem feito exames preventivos e, em ambos os casos, as provas diagnósticas terem apresentado resultados errôneos. "A última que eu fiz, que foi o papanicolau, também fazia a intravaginal, e nada de constatar nada" (US_4_LRS_CaCo_REC).

De acordo com as entrevistadas, problemas de superlotação, dificuldades para 
agendamento e escassez de médicos fazem com que a assistência básica não seja a primeira opção na busca por cuidados para os sintomas do câncer de colo do útero. A maioria dos profissionais entrevistados reforça que a Atenção Básica $(A B)$ não faz nenhum tipo de acompanhamento das usuárias e não recebe contrarreferência dos níveis seguintes.

Em Recife (PE) e Caruaru (PE) houve relatos de erros nos diagnósticos iniciais $\mathrm{e}$ atrasos nos inícios dos tratamentos, com evoluções das doenças, sendo que, em cinco dos seis casos, as mulheres recorreram a exames particulares devido à escassez de vagas públicas nos centros de tratamento e também como forma de receberem os resultados e iniciarem suas terapias mais rapidamente. Não obstante, as entrevistadas também relataram que precisaram de conhecimentos pessoais para acessarem aos serviços de referência, recorrendo à ajuda de conhecidos e de parentes, que facilitaram os acessos iniciais dessas mulheres aos serviços de média e alta complexidade.

Nesse ponto, os discursos das entrevistadas revelaram um dos principais achados desta pesquisa: a cobrança de valores por parte dos médicos para que as pacientes tivessem acesso diferenciado na rede assistencial. A prática foi descrita por usuárias das redes de Caruaru (PE) e Garanhuns (PE), e é conhecida informalmente no sistema de saúde como 'SUS-Plus', sendo SUS a abreviatura de Sistema Único de Saúde e Plus um termo inglês para algo diferenciado, que oferece recursos extras. Neste esquema, as pacientes pagam pelas primeiras consultas com médicos em consultórios particulares e esses mesmos profissionais - que também trabalham na rede pública - facilitam e aceleram não só a entrada dessas mulheres, mas também o acesso a medicamentos e o tratamento através de uma rede de contatos por eles estabelecida nas unidades de referência gratuitas. Essa forma de acesso foi considerada vantajosa para as usuárias que a utilizaram, mas pretere outras mulheres que não fazem a consulta particular, impossibilitando a existência de equidade no acesso.

Eu fiquei desesperada, que a ginecologista disse assim: 'Olhe, esse tratamento de saúde é um tratamento muito caro, muitas pessoas morrem e não têm nem chegado a atender, a passar por esse processo. É difícil, aqui não tem' [...] Foi uma coisa que facilitou pra mim, nesse tratamento, de entrar pelo SUS rápido. Eu achei, assim, que foi mais rápido, devido à consulta que paguei particular. (US_3_LDN_CaCo_GAR).

Após realizar o acesso à rede assistencial, as pacientes declararam que quase todos os exames foram feitos de forma gratuita, ainda que com dificuldades de agendamento nas redes de Recife (PE) e Caruaru (PE). Contudo, em Garanhuns (PE) foi relatada a necessidade de pagar por exames que não estavam disponíveis na rede de saúde. Destaca-se que, nas três redes, pacientes reclamaram, com reforço de seus médicos, da expressiva demora na realização e na entrega de resultados de exames feitos por portadoras de câncer de colo do útero.

Tanto na quimioterapia como na radioterapia, apesar dos incômodos efeitos colaterais, as mulheres declaram que se sentiram satisfeitas com esses serviços e que puderam realizar todas as sessões recomendadas por seus respectivos oncologistas. Não obstante, houve relatos de dificuldades na acessibilidade geográfica para as pacientes que utilizam os serviços fora da capital, em função dos muitos e longos deslocamentos entre uma cidade e outra.

No acesso à medicação, em todos os casos, houve entrega facilitada de medicamentos nos próprios locais onde as pacientes foram tratadas, entretanto, nas três redes ocorreram relatos de que as pacientes não receberam todos os remédios e precisaram comprar alguns para não serem obrigadas a interromper o tratamento.

A análise da continuidade da gestão clínica 
revelou, ainda, que as mulheres não descreveram nenhuma dificuldade significativa na marcação de consultas, na acessibilidade geográfica ou na infraestrutura dos locais de atendimento, após acessarem as unidades de referência.

Em relação à coerência da atenção, foram observadas discordâncias importantes entre os profissionais que atenderam as pacientes nos diferentes serviços dentro das três redes de saúde. Não obstante, profissionais e pacientes apontam que existe coerência entre os objetivos propostos e o tratamento realizado pelos profissionais. Mas, apenas na rede de Recife (PE) houve relatos de que reuniões clínicas aconteciam, enquanto nas demais o tratamento era feito de forma fragmentada, sendo a oncologia o setor diretamente responsável pela paciente. $\mathrm{O}$ estudo evidenciou, ainda, que ocorreu flexibilidade no tratamento das pacientes sempre que foi necessário, de acordo com a particularidade de cada caso.

\section{Continuidade da informação}

No que diz respeito à continuidade da informação, não houve relatos que demonstrassem a existência de conhecimento acumulado entre níveis, em nenhuma das redes. Entretanto, ficou bem caracterizada a deficiência de comunicação entre o nível básico e os serviços especializados, especialmente pela fragilidade no referenciamento entre níveis, pelas falhas nos sistemas de armazenamento de dados das pacientes - que permanecem independentes e isolados -, e pela inexistência ou pouca utilização dos mecanismos de contrarreferência. Em geral, a comunicação acontece de maneira informal entre os profissionais, e a responsabilidade de transmitir as informações para o nível seguinte recai sobre as usuárias, demandando a ocorrência de uma nova anamnese.

É um problema muito grande no SUS. Às vezes, os médicos encaminham o paciente sem dar nenhum detalhe. [...] Eu acho que a informação entre os níveis é absolutamente falha, é um processo absolutamente falho. (US_4_LRS_MédiCo_ACS_CaCo REC).

O estudo apontou, ainda, que o compartilhamento de informações entre diferentes setores dos serviços especializados acontece majoritariamente por meios informais, como telefonemas, bilhetes e e-mails, e, embora tenha sido citado pelos profissionais, não foi percebido pelas usuárias.

Eu sempre mando uma cartinha pra o cirurgião, e normalmente ele manda uma cartinha de resposta. Quando ele não manda uma cartinha, ele me liga pessoalmente e eu anoto no prontuário o que foi conversado. (US_3_LDN_Médica_GMP_CaMa_GAR).

\section{Continuidade da relação}

Quanto à continuidade da relação, o vínculo entre o paciente e o serviço de saúde foi estabelecido apenas no nível especializado, especificamente na oncologia. Em todos os casos, os depoimentos demonstraram que os oncologistas têm interesse pelas pacientes e procuram esclarecer todas as suas dúvidas.

Por fim, no exame da estabilidade e da consistência dos profissionais, o estudo demonstrou que, nas três redes, as pacientes foram muito bem tratadas pelos profissionais do nível especializado, tanto nos hospitais onde fizeram a terapia quanto nas demais unidades por onde passaram.

\section{Discussão}

O estudo realizado apresenta algumas limitações. A primeira é consequência direta do desenho da pesquisa, na qual se utilizou o estudo de caso. Sendo assim, as conclusões e recomendações representam apenas uma aproximação da realidade vivenciada pelas 
mulheres no âmbito das redes por elas utilizadas, o que diminui a amplitude, mas não a importância dos resultados, uma vez que se trata de um estudo exploratório cuja luz das informações pode motivar a realização de outras pesquisas sobre o mesmo tema. Outro problema se refere às informações prestadas pelas mulheres. Dada a gravidade da doença, o foco das mulheres é ter assegurado o seu tratamento e a superação da dor e do sofrimento. Nestas condições, é possível que alguns aspectos relevantes para o tema de estudo não sejam percebidos por elas. Do mesmo modo, há uma limitação da própria capacidade de análise do estudo, pois um dos critérios de seleção da amostra foi que as usuárias já estivessem em tratamento, como forma de avaliar as redes de serviços. Deste modo, seria recomendável estudar os casos de mulheres que não conseguiram acessar aos serviços de referência para o tratamento de câncer de colo do útero. Mesmo assim, foi possível analisar quais são as etapas mais difíceis no decorrer da toda a trajetória feita pelas pacientes nas redes.

Nesse sentido, este estudo vem contribuir com novas informações e entendimentos sobre os diferentes aspectos e dimensões da CA, revelando barreiras e facilidades existentes nas redes de saúde, a partir da percepção das usuárias submetidas ao tratamento para câncer de colo do útero.

$\mathrm{Na}$ análise da continuidade da gestão clínica, evidenciaram-se vários problemas, especialmente no que diz respeito à acessibilidade entre níveis, sobretudo no primeiro nível de atenção. Este achado merece destaque, visto que o câncer do colo do útero é o segundo tumor mais frequente nas mulheres brasileiras (INCA, 2010). A dificuldade de acesso à atenção primária também foi registrada em outros estudos. A maioria coincide no fato de que as barreiras resultam da desmotivação dos usuários para procurar atenção, em razão da burocracia e da demora no tempo de espera entre o agendamento e o dia da consulta, assim como durante o processo de atendimento no serviço. A essas dificuldades, observadas nas três redes, sobretudo em Caruaru (PE), atribuíram-se a falta ou alta rotatividade de profissionais médicos nas Unidades Básicas de Saúde (UBS) (CAmpos ET AL., 2014; GARCIA-SUBIRATS ET AL., 2014A, 2014B; GIOVANELLA ET AL., 2009; VAZQUEZ ET AL., 2013, 2014). Aliados a isto, também chamaram a atenção os erros de diagnósticos e de resultados falso-negativos nos exames realizados antes do início do tratamento, particularmente na rede de Recife (PE). Estudos apontam que o

exame de Papanicolau pode ser usado como um teste de triagem, mas as taxas de resultados falso-negativos são entre $2 \%$ e $50 \%$ dos casos. (INCA, 2012, P. 22).

Todavia, os erros ocorridos em exames não são os únicos motivos para as falhas de diagnóstico, já que, nos protocolos para essa enfermidade, existe a recomendação de que um esfregaço negativo em uma paciente sintomática nunca deve ser considerado como resultado definitivo. Esses elementos podem colaborar para se entender por que, no Brasil, mais da metade dos diagnósticos de câncer de colo do útero são feitos quando o tumor já está na fase de metástase (INCA, 2011).

É importante ressaltar que a falta de acesso a qualquer nível assistencial, em se tratando dessa enfermidade, pode resultar em problemas psicológicos, doenças incapacitantes ou morte para as pacientes, além de gastos evitáveis com consultas, exames, internações em unidades de urgência e de terapia intensiva, ocasionando prejuízos pessoais, familiares, sociais e econômicos significativos. Neste sentido, ações de prevenção do câncer têm sido desenvolvidas no Brasil desde 1984. No entanto, o que se observou foi o descumprimento de normas estabelecidas pelo programa de controle do câncer de colo do útero, lançado em 2011, no qual se encontram diretrizes preconizadas pelo Instituto Nacional do Câncer para a linha de cuidados, com destaque para as ações de controle do 
câncer do colo do útero (CARVALHO; DOMINGOS; LEITE, 2015; INCA, 2010).

Quanto ao acesso aos níveis especializados, a partir das unidades básicas, cinco dos seis casos relataram informalidades e irregularidades na obtenção de vagas nos serviços de referência. Nos últimos cinco anos, vêm sendo observado um aumento no acesso da população brasileira aos serviços básicos de saúde. Mais da metade da população encontra-se cadastrada nas unidades de saúde da família. Contudo, essa expansão da cobertura impulsionada pelo Programa Mais Médicos, criado em 2013, ainda não é suficiente para impactar nas iniquidades de acesso e permitir a continuidade da atenção. Os usuários ainda necessitam da intervenção de amigos, conhecidos e/ou parentes dos profissionais para assegurar atendimento no nível especializado, que, por sua vez, tem limite na capacidade de absorção de demanda (MALTAET AL., 2016; SANTOS; COSTA; GIRARDI, 2015).

A outra forma de acessar aos serviços especializados é através do já citado SUS-Plus. Em três dos quatro casos pesquisados fora da capital, houve relatos de usuárias que tiveram entrada facilitada em toda a estrutura de atendimento gratuita, mediante o pagamento de uma ou mais consultas particulares com médicos que também integram a rede pública. As duas formas de acesso à atenção especializada, ao mesmo tempo em que reduzem o tempo de espera e facilitam o uso, também se configuram como distorções dos procedimentos normatizados e geram graves problemas, como a falta de equidade no acesso de mulheres com câncer ao tratamento adequado na rede pública de saúde. Não foi encontrado nenhum estudo que trate desse tema para efeito de comparação com os resultados que emergiram nesta pesquisa.

Não obstante, o estudo revelou que existe coerência de atenção nas redes de saúde, ainda que ocorram discordâncias entre profissionais; e que também existe flexibilidade, de acordo com as necessidades e possibilidades de cada usuária tratada.
$\mathrm{Na}$ análise da continuidade da informação, não emergiram relatos que revelassem a presença de conhecimento acumulado pelos profissionais nos diferentes níveis nas redes. Entretanto, a transferência da informação não ocorre entre os profissionais de diferentes níveis de atenção, mas apenas entre diferentes setores e unidades do mesmo nível de complexidade. Apenas na rede de Recife (PE) mencionou-se a existência de instrumento de referenciamento, embora seja pouco utilizado. Quando ocorre, a transferência de informação entre os especialistas se dá de maneira predominantemente informal. Esses achados também foram observados em outros estudos sobre o tema (COSTA, 2009; VAZQUEZ ET AL., 2013).

A deficiência na contrarreferência de pacientes com câncer de colo do útero para o nível primário vem sendo justificada pela ausência de um banco de dados integrado, dentro das redes; pela falta de estrutura e preparo das unidades e dos médicos da $\mathrm{AB}$ para fazer o seguimento de mulheres com câncer; e pela cultura assistencial do nível especializado, na qual os oncologistas acompanham e são responsáveis pelas pacientes do início do tratamento até a fase de remissão. Estes resultados são diferentes daqueles encontrados por outros estudos, nos quais os fatores preponderantes foram a "falta de tempo e demora na entrega dos resultados dos exames" (BRITO; OLIVEIRA; SILVA, 2012, P. 418; COSTA, 2009; VAZQUEZ ET AL., 2013). Este fato pode evidenciar que algumas das dificuldades no sistema de referência e contrarreferência dos serviços de alta complexidade podem ser distintas daquelas existentes no tratamento para outras enfermidades que exigem menor nível de complexidade nas redes. Entretanto, para que haja a percepção da continuidade do cuidado, é preciso que ocorra o funcionamento dos serviços em rede, com um sistema institucionalizado de referência e contrarreferência (JULIANI; CIAMPONE, 1999).

Dessa forma, todos os níveis assistenciais são importantes, bem como a qualidade dos registros em cada serviço. 
Identificou-se a existência de vínculos bem estabelecidos entre as usuárias e os médicos que as trataram, porém, isso ocorreu exclusivamente no nível especializado. Alguns autores apontam que as experiências vividas por cada paciente influenciam na visão que eles têm da enfermidade, e na forma como eles vão lidar com a doença e encarar o tratamento (TADDEO ET AL., 2012). O modelo de atenção à saúde fundado na estruturação da assistência em redes pressupõe que a coordenação da atenção é de responsabilidade da AB (LAVRAS, 2011; MENDES, 2011). Entretanto, o estudo apontou que a $\mathrm{AB}$ não é a principal porta de entrada dessas usuárias nas redes de saúde, e nenhuma delas mencionou vínculos ou a responsabilização do profissional da atenção primária pelo seu cuidado. Em todos os casos, as pacientes identificaram o especialista como o responsável pelo seu tratamento. Esses resultados também foram encontrados em um estudo para avaliar a CA no sistema de saúde da Catalunha, Espanha (WAIBEL, 2010).

As entrevistadas declararam, ainda, que os responsáveis por seu tratamento tinham interesse por seus casos e esclareciam todas as suas dúvidas. Outros autores tiveram resultados semelhantes em seus estudos, demonstrando a importância das pacientes receberem informações claras e precisas sobre os seus tratamentos (BRITO; OLIVEIRA; SILVA, 2012; GUSMÃO, 2008; WAIBEL, 2010). Não obstante, estes resultados são discrepantes de outro estudo sobre doenças crônicas, no qual os usuários narraram que os médicos não davam a devida importância às suas necessidades e não mostravam disposição para tirar suas dúvidas, nem esclarecer sobre procedimentos e cuidados no tratamento (TADDEO ET AL., 2012).

Para assegurar o vínculo e a confiança na relação entre profissionais e pacientes, são importantes a estabilidade e a consistência dos profissionais durante o tratamento de doenças crônicas. Nas três redes, as pacientes narraram ter boas relações e confiança nos profissionais que as atenderam; que foram bem tratadas por todas as equipes dos hospitais onde receberam os cuidados que necessitavam; e ressaltaram a importância das consultas terem sido realizadas pelos mesmos profissionais ao longo de suas trajetórias assistenciais. Resultados semelhantes foram encontrados em outros estudos (GULLIFORD; NAITHANI; MORGAN, 2006; NAITHANI; GULLIFORD; MORGAN, 2006; PARKER; CORDEN; HEATON, 2011).

\section{Conclusões e recomendações}

A percepção das mulheres sobre a Continuidade Assistencial no tratamento de câncer de colo do útero evidenciou alguns aspectos de importância para a gestão das redes estudadas. Embora identificassem alguns problemas, as entrevistadas avaliaram de forma positiva a assistência que receberam, após terem acesso aos níveis de média e alta complexidade.

Nas três redes, há descontinuidade na informação que se processa, em geral, através de mecanismos informais de transmissão. Mecanismos normatizados, como guias de referência e resumos de alta, em alguns casos, inexistem ou são subutilizados. Deste modo, os dados sugerem que é necessário investimento para criação de um cadastro único de usuários interligado entre os níveis de atenção, como também para disponibilização e utilização de mecanismos de coordenação assistencial, tais como protocolos, sistemas de referência e contrarreferência, apoio matricial etc.

No entanto, ainda que haja barreiras na rede, os relatos indicaram que as pacientes, na oncologia, têm boa infraestrutura, facilidade na marcação de consultas e formação de vínculo de confiança com os profissionais, o que garante melhores resultados à assistência prestada.

Por fim, os resultados sugerem que, para assegurar a percepção de continuidade de 
tratamento de câncer uterino, são necessários, ainda, investimentos, de modo a garantir a oferta de serviços em rede e de equipes de saúde estáveis, em todos os níveis de atenção, e de forma descentralizada no estado de Pernambuco.

\section{Colaboradores}

Maria Rejane Ferreira da Silva participou do estudo e suas contribuições foram a concepção e o desenho da pesquisa, a obtenção de dados, a análise e interpretação dos dados, a obtenção de financiamento, a redação e a revisão crítica do manuscrito, quanto ao conteúdo intelectual. João Paulo Reis Braga participou do estudo e suas contribuições foram a obtenção de dados, a análise e interpretação dos dados e a redação do manuscrito. José
Fernando do Prado Moura e Jurema Telles de Oliveira Lima participaram do estudo contribuindo na concepção e no desenho da pesquisa, na obtenção de financiamento e na revisão crítica do manuscrito, quanto ao conteúdo intelectual.

\section{Agradecimentos}

Os autores agradecem a participação das mulheres e dos profissionais na pesquisa, como também o apoio financeiro do Ministério da Saúde, do Conselho Nacional de Desenvolvimento Científico e Tecnológico (CNPq), da Fundação de Amparo à Ciência e Tecnologia do Estado de Pernambuco (Facepe) e da Secretaria Estadual de Saúde de Pernambuco (SES/PE), aprovado mediante normas do edital Facepe 13/2012.

\section{Referências}

BARDIN, L. Análise de conteúdo. 3. ed. Lisboa: Edições $70,2009$.

BRITO, E. S. V.; OLIVEIRA, R. C.; SILVA, M. R. F. Análise da continuidade da assistência à saúde de adolescentes portadores de diabetes. Rev. Bras. Saúde Matern. Infant., Recife, v. 12, n. 4, p. 413-423, dez. 2012.

CAMPOS, R. T. O. et al. Avaliação da qualidade do acesso na atenção primária de uma grande cidade brasileira na perspectiva dos usuários. Saúde em Debate, Rio de Janeiro, v. 38, n. esp., p. 252-264, out. 2014.
CARVALHO, B. G.; DOMINGOS, C. M.; LEITE, F. S Integralidade do cuidado no Programa de Controle do Câncer de Colo Uterino: visão das usuárias com alteração na citologia oncótica. Saúde em Debate, Rio de Janeiro, v. 39, n. 106, p. 707-717, set. 2015.

CASTRO GUSMÃO, R. La continuidad asistencial entre niveles asistenciales en pacientes con Enfermedad

Pulmonar Obstructiva Crónica (EPOC). 2008. 99 f. Dissertação (Mestrado em Saúde Pública) Universidade Pompeu Fabra, Barcelona, 2008.

COSTA, R. R. A contra-referência e a continuidade do cuidado dos pacientes hipertensos a partir do serviço 
de emergência do hospital das clínicas de Teresópolis Costantino Ottaviano. 2009. Dissertação (Mestrado em Saúde da Família) - Universidade Estácio de Sá, Rio de Janeiro, 2009.

GARCIA-SUBIRATS, I. et al. Barriers in access to healthcare in countries with different health systems. A cross-sectional study in municipalities of central Colombia and north-eastern Brazil. Soc. Sci. Med., Oxford, v. 106, p. 204-213, abr. 2014a.

GARCIA-SUBIRATS, I. et al. Inequities in access to health care in different health systems: a study in municipalities of central Colombia and north-eastern Brazil. Int. J. Equity Health, Londres, v. 13, n. 1, p. 1-15, jan. 2014b

GIOVANELLA, L. et al. Saúde da família: limites e possibilidades para uma abordagem integral de atenção primária à saúde no Brasil. Ciênc. Saúde Coletiva, Rio de Janeiro, v. 14, n. 3, p. 783-794, jun. 2009.

GULLIFORD, M.; NAITHANI, S.; MORGAN, M. What is "continuity of care"? J. Health Serv. Res. Policy, Edimburgo, v. 11, n. 4, p. 248-250, 2006.

HAGGERTY, J. L. et al. Continuity of care: a multidisciplinary review. BMJ: British Medical Journal, Londres, v. 327, n. 7425, p. 1219-1221, 2003.

\section{INSTITUTO NACIONAL DO CANCER (INCA).}

Diretrizes brasileiras para o rastreamento do câncer do colo do útero. Rio de Janeiro: Ministério da Saúde; INCA, 2011. Disponível em: < http://wwwl.inca.gov.br/ inca/Arquivos/Diretrizes_rastreamento_cancer_colo_ utero.pdf $>$. Acesso em: 7 jun. 2016.

Estimativa 2014: incidência de câncer no Brasil. Rio de Janeiro: Ministério da Saúde; INCA, 2014. Disponível em: <http://www.inca.gov.br/ bvscontrolecancer/publicacoes/Estimativa_2014.pdf $>$. Acesso em: 2 jun. 2016.

Programa Nacional de Controle do Câncer do Colo do Útero. Plano de ação para redução da incidência e mortalidade por câncer do colo do útero. Rio de Janeiro: Ministério da Saúde; INCA, 2010. Disponível em: $<$ http://wwwl.inca.gov.br/inca/Arquivos/sumario_ colo_utero_versao_2011.pdf > . Acesso em: 2 jun. 2016.

JULIANI, C. M. C. M.; CIAMPONE, M. H. T.

Organização do sistema de referência e contrareferência no contexto do sistema único de saúde: a percepção de enfermeiros. Rev. Esc. Enferm. USP, São Paulo, v. 33, n. 4, p. 323-333, 1999.

LAVRAS, C. Atenção primária à saúde e a organização de redes regionais de atenção à saúde no Brasil. Saúde Soc., São Paulo, v. 20, n. 4, p. 867-874, 2011.

MALTA, D. C. et al. A cobertura da Estratégia de Saúde da Família (ESF) no Brasil, segundo a Pesquisa Nacional de Saúde, 2013. Ciênc. Saúde Coletiva, Rio de Janeiro, v. 21, n. 2, p. 327-338, 2016.

MENDES, E. V. As redes de atenção à saúde. 2. ed. Brasilia, DF: Organização Pan-Americana da Saúde (OPAS), 2011.

\section{NAITHANI, S.; GULLIFORD, M.; MORGAN, M.} Patients' perceptions and experiences of "continuity of care" in diabetes. Health Expect, Oxford, v. 9, n. 2, p. 118-129, 2006

PARKER, G.; CORDEN, A.; HEATON, J. Experiences of and influences on continuity of care for service users and carers: synthesis of evidence from a research programme. Health Soc. Care Community, Oxford, v. 19, n. 6, p. 576-601, 2011.

REID, R. J.; HAGGERTY, J.; MCKENDRY, R. Defusing the confusion: concepts and measures of continuity of healthcare. Ottawa: Canadian Health Services Research Foundation (CHSRF), 2002.

SANTOS, L. M. P.; COSTA, A. M.; GIRARDI, S. N. Programa Mais Médicos: uma ação efetiva para reduzir iniquidades em saúde. Ciênc. Saúde Coletiva, Rio de Janeiro, v. 20, n. 11, p. 3547-3552, 2015.

SHORTELL, S. M. et al. Remaking Health Care in America: the evolution of organized delivery systems. 2. ed. São Francisco: The Jossey-Bass Health Care, 2000. 
STEWART, B. W.; WILD, C. P. (Org.). World cancer report 2014. Genebra: WHO, 2014.

TADDEO, P. S. et al. Acesso, prática educativa e empoderamento de pacientes com doenças crônicas. Ciênc. Saúde Coletiva, Rio de Janeiro, v. 17, p. 29232930, 2012.

VAZQUEZ, L. et al. Impact on equity of access and efficiency of Integrated Health care Networks (IHN) in Colombia and Brazil: final report. European Commission: Barcelona, 2013. Disponível em: <http:// www2.equity-la.eu/publicaciones/documentos/ informe-de-resultados-final-2013-equity-la>. Acesso em: 2 ago. 2016.

WAIBEL, S. La continuidad entre niveles asistenciales en dos organizaciones sanitarias integradas de Cataluña desde la perspectiva de los pacientes con enfermedad pulmonar obstructiva crónica. 2010. 92 f. Dissertação (Mestrado em Saúde Pública) - Universidade Pompeu Fabra, Barcelona, 2010.

\section{WORLD HEALTH ORGANIZATION (WHO).}

Globocan 2012: estimated cancer incidence, mortality and prevalence worldwide in 2012. Genebra: WHO, 2012. Disponível em: <http://globocan.iarc.fr $>$. Acesso em: 14 mar. 2015.

Recebido para publicação em fevereiro de 2016

Versão final em junho de 2016

Conflito de interesses: inexistente

Suporte financeiro: Ministério da Saúde (MS), Conselho Nacional de Desenvolvimento Científico e Tecnológico (CNPq), Fundação de Amparo à Ciência e Tecnologia do Estado de Pernambuco (Facepe) e Secretaria Estadual de Saúde de Pernambuco (SES/PE) aprovado mediante normas do edital Facepe 13/2012 